\title{
Nurses and family caregivers of elderly relatives engaged in 4 evolving types of relationships
}

\author{
Ward-Griffin C, McKeever P. Relationships between nurses and family caregivers: partners in care? ANS Adv Nurs Sci \\ 2000 Mar;22:89-103.
QUESTION: What is the nature of the relationships between community nurses and family members caring for elderly relatives?

Design

Qualitative study using a critical ethnographic approach.

\section{Setting \\ Southwestern Ontario, Canada.}

Sources of funding: Health Canada (NHRDP) Research Training Award, Canadian Nurses Foundation Research Grant, and Helen Glass Research Award (Sigma Theta Tau Nursing Society).

For correspondence: Dr C Ward-Griffin Faculty of Health Sciences, University of Western Ontario, London, ON N6A $5 B 8$ Canada.Fax +1519 6613928.

\section{Participants}

23 nurse-family caregiver pairs were identified from 3 community nursing agencies. Most pairs saw each other weekly and had known each other for periods ranging from 3 months to 14 years. All of the family caregivers were women (age range 33-82 y), and most provided care to their husbands who had chronic illnesses.

\section{Methods}

Family caregivers and nurses each participated in private, indepth interviews in which they talked about their experiences of working together. Data from 38 interviews (mean length $75 \mathrm{~min}$ ) and from field notes were transcribed and analysed using, as a frame of refer-

\section{COMMENTARY}

This study by Ward-Griffin and McKeever contributes insights into long term relationships with nurses and the needs of family caregivers who provide chronic care in the home. It identifies potential conflicts in the relationship between nurses and family caregivers. The authors analysed selected findings of a study about home care for the frail elderly. It is unclear how these data fit into the context and goals of the original study. The approach to the analysis and the framework used was well described and methodologically sound.

Clinical implications were examined in the context of family caregiver stress and the effects of conflict. Caregiving is stressful. The addition of relationships that engender chronic tension and conflict increases stress. These chronic stressors, which Lazarus and Folkman describe as daily hassles, have the most negative effects on physical and mental wellbeing and should be avoided or ameliorated. ${ }^{1}$ Interventions to relieve family caregiver stress should be aimed at education, problem solving, social support, and respite. ${ }^{2}$ The findings of Ward-Griffin and McKeever suggest an imbalance in the use of these interventions by nurses. Specifically, nurses underused respite despite caregivers' vocalised needs for relief from the service provision role. Nurses rarely considered increasing their responsibilities or using other less expensive caregiving services to decrease the family caregiver's workload. These strategies were so counterproductive that eventually the caregiver became the patient and the nurse had to provide short term respite services to prevent a crisis. It would be helpful to understand the reason that nurses underused respite as an ongoing intervention to assist family caregivers. Possible explanations are that nurses were unaware of the value of respite, reluctant to refer to less expensive caregiving services, reluctant to relinquish their former role as "hands on" caregiver, unaware of alternate resources, or unable to access additional resources.

Gail Butt, RN, MHSc

Louis Brier Home and Hospital Vancouver, British Columbia, Canada

1 Lazarus R, Folkman S. Stress, appraisal and coping. New York: Springer Publishing, 1984.

2 Zarit SH, Zarit JM. Families under stress: interventions for caregivers of senile dementia patients. Psychotherapy: Theory, Research and Practice 1982:19:461.

ence, Twigg and Atkin's 4 conceptualisations of response of health and social workers to family caregivers.

\section{Main findings}

Nurses and family caregivers had 4 interrelated and evolving types of relationships, each representing different conceptualisations of the roles of the nurse and the family caregiver. In nurse-helper relationships, nurses provided and coordinated care, and family caregivers assumed supportive roles. Each recognised and valued the contributions of the other. Because of the costs of providing chronic care, nurses were expected to shift quickly into the second type of relationship, which was based on the notion of teamwork. In the worker-worker relationship, nurses expected family caregivers to learn the skills necessary to care for the family member. Most caregivers cooperated with nurses to learn these skills; others rejected the premise that they should assume these tasks. Ambiguity about family caregivers' high level of responsibility and low level of authority created tension. Eventually, family caregivers accepted responsibility for all caregiving and moved into the third, and most common type of relationship, the nurse as manager/ family caregiver as worker. With the transfer of caregiving activities to family caregivers, nurses focused on monitoring caregivers' competence and coping skills. Most caregivers were confused and sad that nurses had reduced their time and emotional involvement. They complained that their needs for help with nursing care were not being met. Nurses showed minimal concern for the wellbeing of caregivers. They rarely considered intervening to decrease the caregivers' workload; instead, they advised caregivers to access resources such as caregiver support groups. Because of these discrepancies in values, tension was relatively high. The fourth type of relationship, nurse as nurse/family caregiver as patient, was also common. In this type, family caregivers, who experienced exhaustion and social isolation as a result of the heavy load of caregiving, became the "patients." A source of conflict for nurses was the dual expectation of caring for both the elderly family member and the caregiver. Although nurses expressed concern about the wellbeing of caregivers, they tended to minimise the problems or rationalise that they were limited by the fiscal constraints of the home care system.

\section{Conclusions}

Nurses and family caregivers of elderly relatives oscillated among 4 types of relationships as the caregiving situation evolved. The most common types were nurse as manager/family caregiver as worker and nurse as nurse/caregiver as patient. Contradictory role expectations created tension within these relationships. 